\title{
Development of high-speed capillary spinner cell for in-situ powder diffraction under gas pressure control
}

\author{
S. Kawaguchi', M. Takemoto ${ }^{1}$, K. Sugimoto ${ }^{1,2}$, H. Ashitani ${ }^{3}$, and Y. Kubota ${ }^{3}$ \\ ${ }^{1}$ Japan Synchrotron Radiation Research Institute (JASRI), SPring-8, Sayo, Hyogo, 679-5198, Japan \\ ${ }^{2}$ Institute for Integrated Cell-Material Sciences, Kyoto University, Kyoto 606-8501, Japan, \\ ${ }^{3}$ Department of Physical Science, Osaka Prefecture University, Sakai, Osaka 599 8531, Japan
}

kawaguchi@spring8.or.jp

In-situ synchrotron powder diffraction experiments under a gas atmosphere are one of the most powerful tools used to investigate the crystal structure and to characterize materials function or the applications of gas storage and separation materials. However, in most cases, the information of crystal structures was limited to the static conditions. To understand the overall materials functions to improve thermodynamic and kinetic gas separation properties and storage capacity, it is important to observe continuous structural changes under gas adsorption, desorption and reaction processes. Therefore, we developed new gas handling system [1]. This system can control gas- and vapor-pressure synchronized with the continuous data acquisition of millisecond temporal-resolution highresolution powder diffraction measurements. However, for the high-speed powder diffraction measurement, it is difficult to obtain uniform Debye-Scherrer ring intensity data if the powder sample has large size and/or non-uniform particles. This difficulty is come from an instrumental limitation that the gas cell mounted on diffractometer cannot be rotated during the measurement due to stainless tube to introduce gas to the sample.

To solve this problem, we have developed a new high-speed spinner for in-situ powder diffraction under controlling gas pressure at the beamline BL02B2 at SPring-8 [2]. The high-speed spinner mainly consists of a gas cell to hold the glass capillary with double Orings, a contactless magnetic fluid seal, a brushless motor, and the bearings. The translation and tilt stages are also equipped on the spinner for the alignment of glass capillary sample. The rotation speed can be set to 200 r.p.m. for standard use, and further development of the spinner is currently underway to achieve up to 1000 r.p.m. The various gas species except water and oil are available, and absolute pressure from $1 \mathrm{~Pa}$ to $130 \mathrm{kPa}$ can be controlled.

Using the developed high-speed capillary spinner cell and gas handling system, we have demonstrated in-situ and time-resolved powder diffraction measurements for a nanoporous $\mathrm{Cu}$ coordination polymer [3], which has a pillared layer structure containing onedimensional nanochannels with dimensions of $0.4 \mathrm{~nm} \times 0.6 \mathrm{~nm}$ along the $a$-axis, with large particle size of approximately 20 microns. A two-dimensional flat-panel detector (XRD3025) was used as X-ray detector, and 50 frames of continuous powder diffraction data was obtained in temporal resolution of 0.33 seconds. We tested the evaluation of the diffraction intensity during spinning of capillary sample. On the conditions of high rotation speed with 200 r.p.m., the difference of $5 \%$ between maximum and minimum peak intensity was observed. On the other hand, on the conditions of low rotation speed with 25 r.p.m, twice variation of the peak intensity was observed in diffraction data of 50 frames. In this case, the measurement was performed for a sample with large particles. Moreover, we collected time-resolved diffraction patterns in the Ar gas adsorption process for nanoporous $\mathrm{Cu}$ coordination polymers with different particle sizes of approximately 1, 5, and 20 microns, respectively. As a result, this developed spinner allows to give uniform Debye-Scherrer ring intensity even in sub-second time-resolved data, where the particle size is possibly smaller than that of 5 microns. The results also show that the transition speed from desorption to adsorption phase is highly dependent on the particle size as well as the introduction of gas pressure and temperature. In this presentation, we will show that the mechanism and concept of highspeed capillary spinner cell for in-situ powder diffraction under control of gas atmosphere, and will display the results of the timeresolved powder diffraction measurements for nanoporous $\mathrm{Cu}$ coordination polymers under various $\mathrm{Ar}$ gas adsorption processes.

[1] Kawaguchi, S., Takemoto, M, Tanaka, H., Hiraide, S., Sugimoto, K., \& Kubota, Y. (2020). J. Synchrotron Rad. $27,616-624$.

[2] Kawaguchi, S., Takemoto, M., Osaka, K., Nishibori, E., Moriyoshi, C., Kubota, Y., Kuroiwa, Y. \& Sugimoto, K. (2017). Rev. Sci. Instrum. 88, 085111 .

[3] Kitaura, R., Matsuda, R., Kubota, Y., Kitagawa, S., Tanaka, M., Kobayashi, C. T. \& Suzuki, M. (2005). J. Phys. Chem. B, 109, $23378-23385$.

\section{Keywords: powder diffraction; synchrotron instrumentation; high-speed spinner; gas atmosphere; time-resolved measurement}

This research was supported by KAKENHI Grant Nos. (20H04466, 20H02575, 19KK0132). The synchrotron radiation experiments were performed at beamline BL02B2 of SPring-8 with the approval of the Japan Synchrotron Radiation Research Institute (JASRI) (Proposal Nos. 2019B2094 and 2020A2132). The authors thank Professor K. Otake and Professor S. Kitagawa for their assistance with the preparation of the samples. 